\title{
BMJ Open Treatment of stable chronic obstructive pulmonary disease: protocol for a systematic review and evidence map
}

\author{
Claudia C Dobler, ${ }^{\oplus 1}$ Magdoleen H Farah, ${ }^{1}$ Allison S Morrow, ${ }^{1}$ Mouaz Alsawas, ${ }^{\oplus 1}$ \\ Raed Benkhadra, ${ }^{1}$ Bashar Hasan, ${ }^{1}$ Larry J Prokop, ${ }^{2}$ Zhen Wang, ${ }^{1}$ M Hassan Murad ${ }^{1}$
}

To cite: Dobler CC, Farah MH, Morrow AS, et al. Treatment of stable chronic obstructive pulmonary disease: protocol for a systematic review and evidence map. BMJ Open 2019;9:e027935. doi:10.1136/ bmjopen-2018-027935

- Prepublication history and additional material for this paper are available online. To view these files, please visit the journal online (http://dx.doi. org/10.1136/bmjopen-2018027935).

Received 15 November 2018 Revised 29 January 2019 Accepted 2 April 2019

Check for updates

(c) Author(s) (or their employer(s)) 2019. Re-use permitted under CC BY-NC. No commercial re-use. See rights and permissions. Published by BMJ.

${ }^{1}$ Evidence-Based Practice Center, Robert D. and Patricia

E. Kern Center for the Science of Health Care Delivery, Mayo Clinic, Rochester, Minnesota, USA

${ }^{2}$ Library Public Services, Mayo Clinic, Rochester, Minnesota, USA

Correspondence to Dr Claudia C Dobler; dobler.claudia@mayo.edu; cdobler@bond.edu.au

\section{ABSTRACT}

Introduction Chronic obstructive pulmonary disease (COPD) is a progressive lung disease, usually caused by tobacco smoking, but other important risk factors include exposures to combustion products of biomass fuels and environmental pollution. The introduction of several new (combination) inhaler therapies, increasing uncertainty about the role of inhaled corticosteroids and a rapid proliferation of the literature on management of stable COPD in general, call for novel ways of evidence synthesis in this area. A systematic review and evidence map can provide the basis for shared decision-making tools and help to establish a future research agenda.

Methods and analysis This systematic review will follow an umbrella systematic review design (also called overview of reviews). We plan to conduct a comprehensive literature search of Ovid MEDLINE (including epub ahead of print, in process and other non-indexed citations), Ovid Embase, Ovid Cochrane Database of Systematic Reviews and Scopus from database inception to the present. We will include systematic reviews that assessed the effectiveness of any pharmacological or non-pharmacological intervention on one or more patientimportant outcomes and/or lung function in patients with stable COPD. For every intervention/outcome pair, one systematic review will be included. An a priori protocol will guide, which systematic reviews will be chosen, how their credibility will be evaluated, and how the quality of the body of evidence will be rated. Data will be synthesised into an evidence map that will present a matrix that depicts each available treatment for stable COPD with a quantitative estimate on symptoms/outcomes from the patient perspective, along with an indication of the size and certainty in the evidence.

Ethics and dissemination Approval by a research ethics committee is not required since the review will only include published data. The systematic review will be published in a peer-reviewed journal.

PROSPERO registration number CRD42018095079

\section{INTRODUCTION}

Chronic obstructive pulmonary disease (COPD) is a progressive lung disease characterised by chronic obstruction of airflow and permanent damage to the air sacs that leads to breathing problems. COPD is mainly a consequence of tobacco smoking, but other

\section{Strengths and limitations of this study}

- The planned evidence synthesis will summarise a very large body of the literature on pharmacological and non-pharmacological interventions, thus making research evidence more accessible for stakeholders.

- The systematic review will be the first to use an evidence map to identify evidence gaps and to facilitate evidence communication in clinical encounters for chronic obstructive pulmonary disease.

- For patients and clinicians, the map will facilitate the production of decision aids. For policymakers and researchers, the map helps in establishing a future research agenda.

- The systematic review uses an a priori protocol to identify the most up-to-date systematic reviews of the highest possible quality, and the level of evidence for many intervention/outcome pairs will, therefore, likely be high.

- As we will only include one systematic review per intervention/outcome pair, it is possible that some studies will not have been captured in included systematic reviews.

important risk factors include exposures to combustion products of biomass fuels and environmental pollution. ${ }^{1}$ COPD is the fourth most common cause of death globally and is predicted to be the third by $2030 .^{2}$ In 2010, the number of COPD cases was estimated at 384 million, which corresponded to a global prevalence of $11.7 \% \quad(95 \%$ CI $8.4 \%$ to $15.0 \%){ }^{2}$ COPD was responsible for about $5 \%$ of global disability-adjusted life years $(76.7$ million) and $5 \%$ of total deaths (2.9 million) based on data from the 2010 Global Burden of Disease Study. ${ }^{34}$ In some low-income and middle-income countries COPD has become a growing, but often neglected, epidemic, with a recent study showing a prevalence of COPD of $13.7 \%$ (95\% CI 12.1 to 15.5$)$ in Chinese people aged 40 years or older. ${ }^{5}$ Patients with COPD 
are frequent users of the healthcare system and often need to be admitted to hospital repeatedly, often within short intervals. ${ }^{6}$

Recently, there has been a rapid increase in inhaler therapies available for the management of COPD. In particular, several new inhalers including fixed-dose combinations (containing bronchodilator[s] with or without inhaled corticosteroids) have been introduced. Concurrently, the literature on pharmacological and non-pharmacological interventions for COPD has proliferated substantially. Multiple systematic reviews have been conducted to synthesise the evidence on inhalation treatments in COPD. ${ }^{7-16}$

The role of inhaled corticosteroids for the treatment of stable COPD is increasingly questioned, including in patients with severe disease, driven by the growing evidence of an increased risk of pneumonia associated with inhaled corticosteroids, ${ }^{17} 18$ and the introduction of combined dual long-acting bronchodilator inhalation therapy as a plausible treatment alternative. ${ }^{1920}$

A search of the Cochrane Database of Systematic Reviews (Issue 5 of 12, May 2018) with the keyword 'chronic obstructive pulmonary disease' yielded 132 unique records, indicating that keeping track not just of original studies, but also evidence syntheses, has become a daunting task for decision makers in this area.

The implementation of evidence-based practice in the management of patients with COPD is challenging for medical practitioners due to the rapidly growing body of evidence. Additionally, non-adherence of patients with COPD to prescribed treatments is an ongoing challenge, with many patients being overburdened with the treatment work they need to do for their COPD care. ${ }^{21-24}$ Patients' beliefs and concerns about the safety and benefits of their treatment and complex treatment regimens all impact on adherence. Non-pharmacological interventions, such as pulmonary rehabilitation, are persistently underutilised despite scientific evidence of their effectiveness. ${ }^{25}$

Faced with these challenges, novel tools of evidence synthesis and evidence communication in COPD for the patient-clinician encounter are needed that will allow collaborative deliberation of treatment options between patients and clinicians to make healthcare decisions together, taking into account the best scientific evidence available, as well as the patient's values and preferences. ${ }^{26-29}$ Further, given the rapidly growing body of evidence on treatments for stable COPD, it is timely to identify current knowledge gaps to inform future research needs.

Consequently, the aim of our systematic review is (1) to synthesise the evidence on pharmacological and non-pharmacological treatments in patients with stable COPD and (2) produce an evidence map that identifies evidence gaps in order to inform future research, which provides information that can be incorporated into a decision/communication aid for use during clinical encounters between patients and clinicians.

\section{METHODS AND ANALYSIS}

This protocol adheres to the Preferred Reporting Items for Systematic Review and Meta-analysis Protocols (see online additional file 1). ${ }^{30}$

\section{Patient and public involvement}

No patients or the public were involved in this systematic review of the literature.

\section{Review question}

What are the impacts of pharmacological and non-pharmacological interventions in patients with stable COPD on patient-important outcomes (including dyspnoea and other symptoms, such as anxiety, functional/exercise capacity, frequency of acute exacerbations, health-related quality of life, hospitalisations and emergency department visits) and lung function parameters?

\section{Eligibility criteria}

Types of studies

We will include systematic reviews that assessed the effectiveness of any pharmacological or non-pharmacological intervention on one or more relevant outcomes (see below) in patients with stable COPD. For every intervention/outcome pair (eg, effectiveness of pulmonary rehabilitation to improve dyspnoea), one systematic review will be included. The rationale for including only one systematic review per intervention/outcome pair is to avoid duplication in included original studies. Alternatively, an umbrella review could be conducted at the level of the original studies included in different systematic reviews, which would require a new meta-analysis. The latter approach is significantly more resource-intensive and time-consuming and would not necessarily result in a more accurate pooled estimate compared with the result from a single systematic review, provided that the chosen review is a recent comprehensive high-quality systematic review.

The following a priori protocol will guide, which systematic reviews will be chosen, how their credibility will be evaluated, and how the quality of the body of evidence will be rated.

Systematic reviews will be excluded if:

- Their pooled estimates were (partially) derived from studies that had not been published in the peer-reviewed literature (eg, abstracts and studies only published on pharmaceutical company websites).

- They only contained indirect or mixed indirect and direct comparisons (network meta-analysis).

- They were umbrella reviews (reviews of reviews).

- They included patient populations other than patients with COPD and did not report outcomes separately for patients with COPD.

We will derive a single pooled estimate per outcome per intervention. If multiple systematic reviews provided multiple estimates for the same intervention and outcome, we will choose a systematic review based on the following a priori defined scoring system: 
- Availability of one or more meta-analyses, as opposed to narrative data synthesis only (number of available meta-analyses positively correlated with scores).

- Year of publication and date of literature search (higher scores for more recent systematic reviews).

- Size based on (1) number of studies included and (2) number of participants included (higher scores for greater number of studies and participants).

- Type of studies: randomised controlled trials (RCTs) generally provide stronger evidence than observational studies (highest score for RCTs, followed by prospective cohort studies, retrospective cohort studies and case-control studies).

- Synthesis of data from drug classes rather than specific drugs (higher scores if pooled estimates are available for drug classes compared with specific drugs only).

If we encounter the scenario in which despite the above-mentioned criteria we still have to choose between two or more systematic reviews, we will make this choice based on these additional criteria:

- Consensus among two practising pulmonologists.

- Credibility of the systematic reviews as judged using the 'A Measurement Tool to Assess Systematic Reviews' (AMSTAR2) criteria. $^{31}$

\section{Types of participants}

Studies that evaluated patients aged 40 years and older with stable COPD defined as 'persistent respiratory symptoms and airflow limitations that are due to airway and/ or alveolar abnormalities usually caused by significant exposure to noxious particles or gases ${ }^{, 32}$ will be included. Studies conducted in patients with an acute exacerbation of COPD will be excluded.

\section{Types of interventions}

We will include any pharmacological or non-pharmacological intervention. Interventions that we are expecting are the following: inhaled and other medications, smoking cessation, vaccinations, exercise and pulmonary rehabilitation, airway clearance techniques, nutrition and dietary interventions, COPD action plans, psychological interventions, home oxygen therapy, home mechanical ventilation, interventional bronchoscopy and surgery. Complex interventions with multiple components, such as exercise, smoking cessation advice, psychological support and home visits, will be excluded. We will also exclude interventions only relevant during an acute exacerbation of COPD.

Comparators will include placebo or usual/standard care as well as active interventions. We will exclude indirect comparisons between interventions or mixed direct and indirect comparisons (network meta-analyses).

\section{Types of outcomes}

The following outcomes will be included:

- Dyspnoea and other symptoms (eg, cough, sputum production and fatigue).

- Exercise capacity and functional capacity.
- COPD exacerbations.

- Health-related quality of life.

- Hospitalisations and emergency department visits.

- Mortality.

- Lung function parameters.

- Adverse events.

There will be no restrictions based on measurement methods.

\section{Information sources and search strategy}

This systematic review will follow an umbrella systematic review design (also called overview of reviews). We plan to conduct a comprehensive literature search of six databases, including Ovid MEDLINE epub ahead of print, Ovid MEDLINE in process and other non-indexed citations, Ovid MEDLINE, Ovid Embase, Ovid Cochrane Database of Systematic Reviews and Scopus from database inception to the present. We have developed a preliminary database search strategy and found that these databases can adequately identify the relevant literature. Reference mining of relevant publications will be conducted. The search strategy will be designed and conducted by an experienced librarian with input from the study's principal investigator. Controlled vocabulary supplemented with keywords will be used to search for systematic reviews and meta-analyses of pharmacological and non-pharmacological treatments for stable COPD. Search strategies are shown in online additional file 2.

All citations identified through the process will be imported to a reference management system (EndNote V.X7 and V.X8; Thomson Reuters, Philadelphia, PA, USA). We will use a web-based systematic review software, DistillerSR (Evidence Partners Incorporated, Ottawa, Canada), to facilitate the study selection process.

\section{Data extraction}

For every intervention/outcome pair, one systematic review will be chosen following the priori protocol outlined above. At the beginning of data abstraction, we will develop a standardised data extraction form to extract study characteristics (author, study design, inclusion and exclusion criteria, patient characteristics, interventions, comparisons, outcomes and related items for assessing study quality and applicability). The standardised form will be pilot-tested by all study team members. We will iteratively continue testing the form until no additional items or unresolved questions exist. All study details will be extracted by two independent reviewers. A third reviewer will review data extraction, and resolve conflicts.

\section{Strategy for data synthesis}

Data will be synthesised into an evidence map. An evidence map is defined as a systematic search of a broad field to identify gaps in knowledge and/or future research needs that presents results in a user-friendly format, often a visual figure or graph, or a searchable database. ${ }^{33}$ The planned map will present a matrix that depicts each available treatment for stable COPD with a 
quantitative estimate on symptoms/outcomes from the patient perspective, along with an indication of the size and certainty in the evidence.

We will also provide a narrative synthesis of the findings from the included systematic reviews, structured around the type of intervention, target population characteristics (eg, severity of COPD), type of outcome and intervention content.

\section{Analysis of subgroups or subsets}

Predetermined characteristics for subgroup analysis are:

- Severity of COPD, for example, based on Global Initiative for Chronic Obstructive Lung Disease criteria, ${ }^{34}$ forced expiratory volume in $1 \mathrm{~s}$ in per cent predicted.

- COPD phenotypes, for example, patients with frequent exacerbations, eosinophilic inflammation and emphysema-hyperinflation. ${ }^{35}$

- Duration of intervention.

- Different study types (eg, RCTs vs observational studies).

\section{Credibility (methodological quality) assessment}

We will use AMSTAR2 ${ }^{31}$ to assess the credibility of the included systematic reviews. The AMSTAR2 tool addresses the following 16 items:

- Use of the components of population, intervention, comparator and outcome for research questions and inclusion criteria.

- Protocol for the systematic review, justification of any significant protocol deviations.

- Study selection.

- Literature search strategy.

- Study selection by two independent reviewers.

- Data extraction by two independent reviewers.

- Excluded studies.

- Description of included studies.

- Risk of bias assessment in individual studies.

- Sources of funding.

- Methods for meta-analysis.

- Impact of the risk of bias on the meta-analysis or other evidence syntheses.

- Accounting for risk of bias in the interpretation/ discussion of results.

- Explanation for heterogeneity in the results.

- Publication bias.

- Conflicts of interest.

\section{ETHICS AND DISSEMINATION}

This systematic review is registered with PROSPERO (http://www.crd.york.ac.uk/PROSPERO). Important protocol amendments will be documented in PROSPERO. Approval by a research ethics committee is not required since the review will only include published and publicly accessible data. The systematic review will be published in a peer-reviewed journal and will provide various stakeholders with an evidence map.

\section{DISCUSSION}

The aim of this systematic review is to systematically identify, summarise and assess a large body of evidence on pharmacological and non-pharmacological interventions in stable COPD. The information will be used to produce an evidence map to identify knowledge gaps and to inform a decision/communication aid for the clinical encounter between patients and clinicians.

\section{Strengths and limitations of this systematic review}

We have not identified any systematic reviews that have provided an evidence map for the treatment of stable COPD, and this systematic review will, therefore, be the first to use an evidence map to identify evidence gaps and to facilitate evidence communication in clinical encounters for COPD. The systematic review uses an a priori protocol to identify the most up-to-date systematic reviews of the highest possible quality, and the level of evidence for many intervention/outcome pairs will, therefore, likely be high.

As we will only include one systematic review per intervention/outcome pair, it is possible that some studies will not have been captured in included systematic reviews.

\section{Practical implications}

Evidence mapping is a relative novel method of evidence synthesis, which aims to identify gaps in knowledge and/ or future research needs based on a comprehensive literature search and present results in an easy to understand format in a figure or graph. ${ }^{33}$ Evidence presented in such a user-friendly way may facilitate knowledge dissemination and implementation among relevant stakeholder groups including policymakers. ${ }^{36}{ }^{37}$ In this proposed study, we are targeting two aims that address the needs of different stakeholders.

The first type of stakeholders is patients and clinicians. In the context of a clinical encounter, they require shared decision-making tools (decision aids) because the available treatments are numerous and the impact on the different symptoms varies by intervention. Traditional systematic reviews usually summarise evidence grouped around specific interventions. In clinical practice, however, discussions between patients and clinicians often focus on a problem that demands a solution (eg, shortness of breath or limited functional capacity in patients with COPD). This requires that evidence is communicated from an outcome rather than an intervention perspective (eg, which interventions can improve shortness of breath or functional capacity in patients with stable COPD?). We plan to present the results of our systematic review structured by outcomes to facilitate knowledge translation into a decision/communication aid (the COPD Choice decision-aid project). This will, hopefully, contribute to patient-centred and transparent evidence communication in clinical encounters.

The second type of stakeholders is policymakers funding research and researchers. The presentation of results in the form of an evidence map can quickly provide them 
with a snapshot of which symptoms (daily dilemmas that patients face) or which interventions are only supported by low-quality evidence (or no evidence). Such areas are prime targets for future research.

The proposed approach (overview of reviews and evidence mapping) is ideal in the context of the very large volume of the literature available on pharmacological and non-pharmacological interventions in stable COPD, and the need to synthesise and present summaries that cater to different stakeholders.

Contributors CCD drafted the manuscript. MHM made substantial contributions to conception and design. Working with CCD, LJP designed the search strategy for the systematic review. CCD, MHF, ASM, MA, RB, BH, LJP, ZW and MHM revised the manuscript critically for important intellectual content and approved the final manuscript.

Funding This research received no specific grant from any funding agency in the public, commercial or not-for-profit sectors. CCD was supported by a fellowship from the Australian National Health and Medical Research Council (NHMRC), grant number APP1123733.

Competing interests None declared.

Patient consent for publication Not required.

Provenance and peer review Not commissioned; externally peer reviewed.

Open access This is an open access article distributed in accordance with the Creative Commons Attribution Non Commercial (CC BY-NC 4.0) license, which permits others to distribute, remix, adapt, build upon this work non-commercially, and license their derivative works on different terms, provided the original work is properly cited, appropriate credit is given, any changes made indicated, and the use is non-commercial. See: http://creativecommons.org/licenses/by-nc/4.0/.

\section{REFERENCES}

1. Postma DS, Bush A, van den Berge M. Risk factors and early origins of chronic obstructive pulmonary disease. Lancet 2015;385:899-909.

2. Adeloye D, Chua S, Lee $C$, et al. Global and regional estimates of COPD prevalence: Systematic review and meta-analysis. J Glob Health 2015;5:020415.

3. Murray CJ, Vos T, Lozano R, et al. Disability-adjusted life years (DALYs) for 291 diseases and injuries in 21 regions, 1990-2010: a systematic analysis for the Global Burden of Disease Study 2010. Lancet 2012;380:2197-223.

4. Lozano R, Naghavi M, Foreman K, et al. Global and regional mortality from 235 causes of death for 20 age groups in 1990 and 2010: a systematic analysis for the Global Burden of Disease Study 2010. Lancet 2012;380:2095-128.

5. Wang $\mathrm{C}, \mathrm{Xu} J$, Yang L, et al. Prevalence and risk factors of chronic obstructive pulmonary disease in China (the China Pulmonary Health $[\mathrm{CPH}]$ study): a national cross-sectional study. Lancet 2018;391:1706-17.

6. Hakim MA, Garden FL, Jennings MD, et al. Performance of the LACE index to predict 30-day hospital readmissions in patients with chronic obstructive pulmonary disease. Clin Epidemiol 2018;10:51-9.

7. Horita N, Goto A, Shibata Y, et al. Long-acting muscarinic antagonist (LAMA) plus long-acting beta-agonist (LABA) versus LABA plus inhaled corticosteroid (ICS) for stable chronic obstructive pulmonary disease (COPD). Cochrane Database Syst Rev 2017;2:CD012066.

8. Calzetta L, Ora J, Cavalli F, et al. Impact of LABA/LAMA combination on exercise endurance and lung hyperinflation in COPD: A pair-wise and network meta-analysis. Respir Med 2017;129:189-98.

9. Farne HA, Cates CJ. Long-acting beta2-agonist in addition to tiotropium versus either tiotropium or long-acting beta2-agonist alone for chronic obstructive pulmonary disease. Cochrane Database Syst Rev 2015:CD008989.

10. Rodrigo GJ, Price D, Anzueto A, et al. LABA/LAMA combinations versus LAMA monotherapy or LABA/ICS in COPD: a systematic review and meta-analysis. Int J Chron Obstruct Pulmon Dis 2017;12:907-22

11. Karner C, Cates CJ. Combination inhaled steroid and long-acting beta(2)-agonist in addition to tiotropium versus tiotropium or combination alone for chronic obstructive pulmonary disease. Cochrane Database Syst Rev 2011:CD008532.
12. Kwak MS, Kim E, Jang EJ, et al. The efficacy and safety of triple inhaled treatment in patients with chronic obstructive pulmonary disease: a systematic review and meta-analysis using Bayesian methods. Int J Chron Obstruct Pulmon Dis 2015;10:2365-76.

13. Nannini LJ, Lasserson TJ, Poole P. Combined corticosteroid and long-acting beta(2)-agonist in one inhaler versus long-acting beta(2)agonists for chronic obstructive pulmonary disease. Cochrane Database Syst Rev 2012:CD006829.

14. Nannini LJ, Poole P, Milan SJ, et al. Combined corticosteroid and long-acting beta(2)-agonist in one inhaler versus inhaled corticosteroids alone for chronic obstructive pulmonary disease. Cochrane Database Syst Rev 2013:CD006826.

15. Rojas-Reyes MX, García Morales OM, Dennis RJ, et al. Combination inhaled steroid and long-acting beta ${ }_{2}$-agonist in addition to tiotropium versus tiotropium or combination alone for chronic obstructive pulmonary disease. Cochrane Database Syst Rev 2016:CD008532.

16. Welsh EJ, Cates CJ, Poole P. Combination inhaled steroid and long-acting beta2-agonist versus tiotropium for chronic obstructive pulmonary disease. Cochrane Database Syst Rev 2013:CD007891.

17. Singh S, Amin AV, Loke YK. Long-term use of inhaled corticosteroids and the risk of pneumonia in chronic obstructive pulmonary disease: a meta-analysis. Arch Intern Med 2009;169:219-29.

18. Kew KM, Seniukovich A. Inhaled steroids and risk of pneumonia for chronic obstructive pulmonary disease. Cochrane Database Syst Rev 2014:CD010115.

19. Wedzicha JA, Banerji D, Chapman KR, et al. IndacaterolGlycopyrronium versus Salmeterol-Fluticasone for COPD. N Engl J Med 2016;374:2222-34.

20. Magnussen H, Disse B, Rodriguez-Roisin R, et al. Withdrawal of inhaled glucocorticoids and exacerbations of COPD. N Engl J Med 2014;371:1285-94

21. Bourbeau J, Bartlett SJ. Patient adherence in COPD. Thorax 2008;63:831-8.

22. Ágh T, Inotai A, Mészáros Á. Factors associated with medication adherence in patients with chronic obstructive pulmonary disease. Respiration 2011;82:328-34.

23. Harb N, Foster J, Dobler C. Patient-perceived treatment burden of chronic obstructive pulmonary disease. Int J Chron Obstruct Pulmon Dis 2017;12:1641-52.

24. Dobler CC, Harb N, Maguire CA, et al. Treatment burden should be included in clinical practice guidelines. BMJ 2018;363:k4065.

25. Safka KA, Mclvor RA. Non-pharmacological management of chronic obstructive pulmonary disease. The Ulster medical journal 2015;84:13-21.

26. Stiggelbout AM, Van der Weijden T, De Wit MP, et al. Shared decision making: really putting patients at the centre of healthcare. BMJ 2012;344:e256.

27. Elwyn G, Frosch D, Thomson R, et al. Shared Decision Making: A Model for Clinical Practice. J Gen Intern Med 2012;27:1361-7.

28. Dobler CC, Sanchez M, Gionfriddo MR, et al. Impact of decision aids used during clinical encounters on clinician outcomes and consultation length: a systematic review. BMJ Qual Saf 2018:bmjqs-2018-008022 (published Online First: 12 Oct 2018).

29. Dobler CC, Midthun DE, Montori VM. Quality of Shared Decision Making in Lung Cancer Screening: The Right Process, With the Right Partners, at the Right Time and Place. Mayo Clin Proc 2017;92:1612-6.

30. Shamseer L, Moher D, Clarke M, et al. Preferred reporting items for systematic review and meta-analysis protocols (PRISMA-P) 2015: elaboration and explanation. BMJ 2015;349:g7647.

31. Shea BJ, Reeves BC, Wells G, et al. AMSTAR 2: a critical appraisal tool for systematic reviews that include randomised or nonrandomised studies of healthcare interventions, or both. BMJ 2017;358:j4008.

32. Global Initiative for Chronic Obstructive Lung Disease. Global strategy for the diagnosis, management, and prevention of chronic obstructive pulmonary disease. 2019. report https://goldcopd.org/ gold-reports/ (accessed January 28, 2019).

33. Miake-Lye IM, Hempel S, Shanman R, et al. What is an evidence map? A systematic review of published evidence maps and their definitions, methods, and products. Syst Rev 2016;5:28.

34. Global Strategy for Prevention, Diagnosis and Management of COPD, 2018 reprt. http://goldcopd.org/wp-content/uploads/2017/ 11/GOLD-2018-v6.0-FINAL-revised-20-Nov_WMS.pdf (Accessed 15 May 2018).

35. Miravitlles M, Calle M, Soler-Cataluña JJ. Clinical phenotypes of COPD: identification, definition and implications for guidelines. Arch Bronconeumol 2012;48:86-98. 
36. Bangerter LR, Griffin JM, Langer S, et al. The effect of psychosocial interventions on outcomes for caregivers of hematopoietic cell transplant patients. Curr Hematol Malig Rep 2018;13:155-63.
37. Farah WH, Alsawas M, Mainou M, et al. Non-pharmacological treatment of depression: a systematic review and evidence map. Evid Based Med 2016;21:214-21. 